\title{
A characterization of evolution operators
}

\author{
by \\ NAOKI TANAKA (Okayama)
}

\begin{abstract}
A class of evolution operators is introduced according to the device of Kato. An evolution operator introduced here provides a classical solution of the linear equation $u^{\prime}(t)=A(t) u(t)$ for $t \in[0, T]$, in a general Banach space. The paper presents a necessary and sufficient condition for the existence and uniqueness of such an evolution operator.
\end{abstract}

Introduction. Throughout this paper, let $X$ and $Y$ be real Banach spaces such that $Y$ is densely and continuously embedded in $X$. After a pioneering work on "hyperbolic" equations by Kato [5], many authors investigated sufficient conditions for the existence of an evolution operator on $X$ which provides a classical solution of the linear evolution equation

$$
u^{\prime}(t)=A(t) u(t) \quad \text { for } t \in[0, T],
$$

where $\{A(t): t \in[0, T]\}$ is a family of closed linear operators in $X$ satisfying the following condition:

(A) For $t \in[0, T], D(A(t)) \supset Y$ and $A(t)$ is strongly continuous on $[0, T]$ in $B(Y, X)$.

Among others, Kobayasi [7] obtained a fundamental and important generation theorem of evolution operators under the stability condition in the sense of Kato. For some related results we refer to the papers by Dorroh [1], Ishii [4], Kato [6], and Yagi [12] and the books by Goldstein [2], Pazy [9] and Tanabe [10].

Recently, the author [11] has proposed a new stability condition from the viewpoint of finite difference approximations, and showed that evolution operators can be generated even if the new stability condition is assumed instead of Kato's stability condition. So far sufficient conditions for the existence of evolution operators associated with (E) have been investigated extensively. A Hille-Yosida type theory for evolutions was discussed by Kōmura [8] and Herod and McKelvey [3] (see also the references cited

2000 Mathematics Subject Classification: Primary 47D06; Secondary 34G10. 
therein), but it seems to the author that there is no characterization for the Kato class of evolution operators.

We are interested in finding a necessary and sufficient condition for the existence and uniqueness of an evolution operator introduced by Kato. In Section 1, we introduce a class of evolution operators which provide us with classical solutions of (E), according to the device of Kato [6], and give a necessary condition for such evolution operators (which are called solution operators) to exist (Proposition 1.2). Section 2 contains the construction of approximate solutions for (E) which is used to get an approximation of the desired evolution operator. Section 3 presents the main theorem (Theorem 3.1 ) and an extension of the main results of $[6,11]$ (Corollary 3.3).

1. A class of evolution operators. Throughout this paper, we use another Banach space $Z$ and a norm continuous family $\{S(t): t \in[0, T]\}$ in $B(Y, Z)$ satisfying the following condition:

There exist $C_{S} \geq c_{S}>0$ such that

$$
c_{S}\|u\|_{Y} \leq\|u\|_{X}+\|S(t) u\|_{Z} \leq C_{S}\|u\|_{Y}
$$

for $u \in Y$ and $t \in[0, T]$.

Moreover, we assume that there exists a strongly continuous nonexpansive homomorphism $\Xi$ of the algebra $B(X)$ into $B(Z)$; that is, $\Xi$ is an algebraic homomorphism such that $\Xi I=I_{Z},\|\Xi B\|_{Z} \leq\|B\|_{X}$ for $B \in B(X)$, and if $\left\{B_{n}\right\}$ is a sequence in $B(X)$, then $\lim _{n \rightarrow \infty} B_{n} x=B x$ for $x \in X$ implies $\lim _{n \rightarrow \infty}\left(\Xi B_{n}\right) z=(\Xi B) z$ for $z \in Z$.

An evolution operator $\{U(t, s)\}$ in $B(X)$ defined on the triangle

$$
\Delta=\{(t, s): 0 \leq s \leq t \leq T\}
$$

satisfying the following properties was introduced implicitly in Kato's paper $[6]$ :

(E3) $U(t, s)(Y) \subset Y$ for $(t, s) \in \Delta$, and $U(t, s)$ is strongly continuous on $\Delta$ in $B(Y)$.

(E4) For $(s, y) \in[0, T) \times Y, U(t, s) y$ is differentiable in $t \in[s, T]$, and

$$
(\partial / \partial t) U(t, s) y=A(t) U(t, s) y \quad \text { for }(t, s) \in \Delta \text { and } y \in Y .
$$

(E5) The integral equality

$$
S(t) U(t, s) y=\Xi U(t, s) S(s) y+\int_{s}^{t} \Xi U(t, \sigma)(B(\sigma) S(\sigma)+C(\sigma)) U(\sigma, s) y d \sigma
$$


holds for $(t, s) \in \Delta$ and $y \in Y$, where $\{B(t): t \in[0, T]\}$ is a strongly continuous family in $B(Z)$ and $\{C(t): t \in[0, T]\}$ is a strongly continuous family in $B(X, Z)$.

Proposition 1.1. An evolution operator satisfying (E1) through (E5) is uniquely determined by $\{A(t): t \in[0, T]\}$.

In this paper, an evolution operator satisfying conditions (E1) through (E5) is called a solution operator governed by $\{A(t): t \in[0, T]\}$.

Proof of Proposition 1.1. Let $y \in Y$ and $(t, s) \in \Delta$ with $t \neq s$. Then we have, for $h>0$ with $(t, s+h) \in \Delta$,

$$
(U(t, s+h) y-U(t, s) y) / h=U(t, s+h)(y-U(s+h, s) y) / h,
$$

and as $h \downarrow 0$ the right-hand side tends to $-U(t, s) A(s) y$, by condition (E4). Since $U(t, s) A(s) y$ is continuous on $\Delta$ in $X$ by condition (E2), we see that for $(t, y) \in(0, T] \times Y, U(t, s) y$ is differentiable in $s \in[0, t]$ and $(\partial / \partial s) U(t, s) y=$ $-U(t, s) A(s) y$ for $(t, s) \in \Delta$ and $y \in Y$.

Now, let $\{V(t, s):(t, s) \in \Delta\}$ be another evolution operator on $X$ satisfying (E1) through (E5) with $U(t, s)$ replaced by $V(t, s)$. Then we have, by the fact shown above and (E4),

$$
(\partial / \partial \sigma) U(t, \sigma) V(\sigma, s) y=U(t, \sigma)(-A(\sigma)+A(\sigma)) V(\sigma, s) y=0
$$

for $(t, s) \in \Delta$ and $y \in Y$; hence $U(t, s)=V(t, s)$ for $(t, s) \in \Delta$, because $Y$ is dense in $X$.

The purpose of this section is to investigate some properties deduced from conditions (E1) through (E5).

Proposition 1.2. Let $\{U(t, s):(t, s) \in \Delta\}$ be a solution operator governed by $\{A(t): t \in[0, T]\}$ such that $\|U(t, s)\|_{X} \leq M$ for $(t, s) \in \Delta$. Then there exist $\varepsilon_{0}>0$ and a family $\{J(t, s)\}$ in $B(X)$ defined on the set $\Delta_{0}=$ $\left\{(t, s) \in \Delta:|t-s| \leq \varepsilon_{0}\right\}$ such that the following conditions are satisfied:

(A1) The estimate

$$
\left\|\prod_{i=1}^{k} J\left(t_{i}, t_{i-1}\right)\right\|_{X} \leq M
$$

holds for every finite sequence $\left\{t_{i}\right\}_{i=0}^{k}$ such that $\left(t_{i}, t_{i-1}\right) \in \Delta_{0}$ for $i=1, \ldots, k$.

(A2) $\quad J(t, s)(Y) \subset Y$ for $(t, s) \in \Delta_{0}$, and $J(t, s)$ is strongly continuous on $\Delta_{0}$ in $B(Y)$.

(A3) There exists a strongly continuous family $\left\{E_{1}(t, s):(t, s) \in \Delta_{0}\right\}$ in $B(Y, X)$ satisfying $E_{1}(t, t)=0$ for $t \in[0, T]$ such that

$$
J(t, s) y=y+(t-s) A(t) J(t, s) y+(t-s) E_{1}(t, s) y
$$

for $(t, s) \in \Delta_{0}$ and $y \in Y$. 
(A4) There exists a strongly continuous family $\left\{E_{2}(t, s):(t, s) \in \Delta_{0}\right\}$ in $B(Y, Z)$ satisfying $E_{2}(t, t)=0$ for $t \in[0, T]$ such that

$$
\begin{aligned}
S(t) J(t, s) y= & \Xi J(t, s) S(s) y+(t-s)(B(t) S(t)+C(t)) J(t, s) y \\
& +(t-s) E_{2}(t, s) y
\end{aligned}
$$

for $(t, s) \in \Delta_{0}$ and $y \in Y$.

Proof. Set $\varepsilon_{0}=T$ and $\Delta_{0}=\Delta$, and define $J(t, s)=U(t, s)$ for $(t, s) \in$ $\Delta_{0}$. To check (A1), let $\left\{t_{i}\right\}_{i=0}^{k}$ be such that $\left(t_{i}, t_{i-1}\right) \in \Delta_{0}$ for $i=1, \ldots, k$. By condition (E1) we have $\prod_{i=1}^{k} J\left(t_{i}, t_{i-1}\right)=J\left(t_{k}, t_{0}\right)$, from which condition (A1) is deduced. Property (A2) is a direct consequence of (E3). Define $E_{1}$ : $\Delta_{0} \rightarrow B(Y, X)$ by

$$
E_{1}(t, s) y=\frac{1}{t-s} \int_{s}^{t}(A(r) U(r, s) y-A(t) U(t, s) y) d r
$$

for $(t, s) \in \Delta_{0}$ with $t \neq s$, and $E_{1}(t, t)=0$ for $t \in[0, T]$. Property (A3) follows easily from condition (E4). By condition (E5), a simple computation yields that (A4) is satisfied with $E_{2}: \Delta_{0} \rightarrow B(Y, Z)$ defined by

$$
\begin{aligned}
E_{2}(t, s) y=\frac{1}{t-s} \int_{s}^{t}(\Xi U(t, r)(B(r) S(r) & +C(r)) U(r, s) y \\
& -(B(t) S(t)+C(t)) U(t, s) y) d r
\end{aligned}
$$

for $(t, s) \in \Delta_{0}$ with $t \neq s$, and $E_{2}(t, t)=0$ for $t \in[0, T]$.

Proposition 1.3. Assume that $J(t, t)=I$ for $t \in[0, T]$. Then condition (A1) is equivalent to the following condition:

(a1) There exists a family $\left\{N_{t}(\cdot): t \in[0, T]\right\}$ of norms in $X$ such that

(i) $\|x\|_{X} \leq N_{t}(x) \leq M\|x\|_{X}$ for $x \in X$ and $t \in[0, T]$,

(ii) $N_{t}(J(t, s) x) \leq N_{s}(x)$ for $x \in X$ and $(t, s) \in \Delta_{0}$.

Proof. Assume that (a1) is satisfied, and let $\left\{t_{i}\right\}_{i=0}^{k}$ be such that $\left(t_{i}, t_{i-1}\right)$ $\in \Delta_{0}$ for $i=1, \ldots, k$. By (ii) we have $N_{t_{i}}\left(J\left(t_{i}, t_{i-1}\right) x\right) \leq N_{t_{i-1}}(x)$ for $x \in X$ and $i=1, \ldots, k$, and so $N_{t_{k}}\left(\prod_{i=1}^{k} J\left(t_{i}, t_{i-1}\right) x\right) \leq N_{t_{0}}(x)$ for $x \in X$. This fact and (i) together imply condition (A1).

Conversely, assume that (A1) holds, and for each $t \in[0, T]$ define a norm $N_{t}(\cdot)$ in $X$ by

$$
N_{t}(x)=\sup \left\{\left\|\prod_{i=1}^{k} J\left(t_{i}, t_{i-1}\right) x\right\|_{X}\right\}
$$

where the supremum is taken over all finite sequences $\left\{t_{i}\right\}_{i=0}^{k}$ such that $t=t_{0} \leq t_{1} \leq \ldots \leq t_{k} \leq T$ and $\left(t_{i}, t_{i-1}\right) \in \Delta_{0}$ for $i=1, \ldots, k$. Let $t \in[0, T]$ and consider the sequence $\left\{t_{i}\right\}_{i=0}^{k}$ with $t_{i}=t$ for $i=0,1, \ldots, k$. 
Then $\prod_{i=0}^{k} J\left(t_{i}, t_{i-1}\right)=I$, because $J(t, t)=I$ for $t \in[0, T]$. This implies that $\|x\|_{X} \leq N_{t}(x)$ for $x \in X$. The fact that $N_{t}(x) \leq M\|x\|_{X}$ for $x \in X$ is deduced easily from condition (A1).

To check (ii), let $x \in X$ and $(t, s) \in \Delta_{0}$. For a finite sequence $\left\{t_{i}\right\}_{i=0}^{k}$ with $t=t_{0} \leq t_{1} \leq \ldots \leq t_{k} \leq T$ and $\left(t_{i}, t_{i-1}\right) \in \Delta_{0}$ for $i=1, \ldots, k$, the sequence $\left\{s_{l}\right\}_{l=0}^{k+1}$, defined by $s_{0}=s$ and $s_{l}=t_{l-1}$ for $l=1, \ldots, k+1$, satisfies $s=s_{0} \leq s_{1} \leq \ldots \leq s_{k+1} \leq T$ and $\left(s_{l}, s_{l-1}\right) \in \Delta_{0}$ for $l=1, \ldots, k+1$. By the definition of $N_{s}(\cdot)$, we have

$$
\left\|\prod_{i=1}^{k} J\left(t_{i}, t_{i-1}\right) J(t, s) x\right\|_{X}=\left\|\prod_{l=1}^{k+1} J\left(s_{l}, s_{l-1}\right) x\right\|_{X} \leq N_{s}(x) ;
$$

hence $N_{t}(J(t, s) x) \leq N_{s}(x)$ for $x \in X$.

Proposition 1.4. Assume that condition (A1) is satisfied and $J(t, s)(Y)$ $\subset Y$ for $(t, s) \in \Delta_{0}$. Then conditions (A2) through (A4) hold if and only if the following two conditions are satisfied:

(i) There exists a strongly continuous family $\left\{E_{3}(t, s):(t, s) \in \Delta_{0}\right\}$ in $B(Y, X)$ satisfying $E_{3}(t, t)=0$ for $t \in[0, T]$ such that

$$
J(t, s) y=y+(t-s) A(s) y+(t-s) E_{3}(t, s) y
$$

for $(t, s) \in \Delta_{0}$ and $y \in Y$.

(ii) There exists a strongly continuous family $\left\{E_{4}(t, s):(t, s) \in \Delta_{0}\right\}$ in $B(Y, Z)$ satisfying $E_{4}(t, t)=0$ for $t \in[0, T]$ such that

$$
\begin{aligned}
S(t) J(t, s) y= & \Xi J(t, s) S(s) y+(t-s) \Xi J(t, s)(B(s) S(s)+C(s)) y \\
& +(t-s) E_{4}(t, s) y
\end{aligned}
$$

for $(t, s) \in \Delta_{0}$ and $y \in Y$.

Proof. Assume that conditions (A2) through (A4) are satisfied, and set

$$
E_{3}(t, s) y=E_{1}(t, s) y-A(s) y+A(t) J(t, s) y
$$

for $(t, s) \in \Delta_{0}$ and $y \in Y$. Equation (1.1) with $t=s$ implies $J(t, t)=I$ for $t \in[0, T]$. Clearly, $E_{3}(t, t)=0$ for $t \in[0, T]$, and the strong continuity of $E_{3}(t, s)$ on $\Delta_{0}$ in $B(Y, X)$ is deduced from condition (A2). By condition (A3) we have (1.3). To prove (ii), set

$$
E_{4}(t, s) y=(B(t) S(t)+C(t)) J(t, s) y-\Xi J(t, s)(B(s) S(s)+C(s)) y+E_{2}(t, s) y
$$

for $(t, s) \in \Delta_{0}$ and $y \in Y$. Equation (1.4) is clearly satisfied. By condition (A1) and the density of $Y$ in $X$ we deduce from (A2) that $J(t, s)$ is strongly continuous on $\Delta_{0}$ in $B(X)$. This fact and the strong continuity of $\Xi$ together imply the strong continuity of $\Xi J(t, s)$ on $\Delta_{0}$ in $B(Z)$. It follows that $E_{4}(t, s)$ is strongly continuous on $\Delta_{0}$ in $B(Y, Z)$. 
Conversely, assume that (i) and (ii) are satisfied. The strong continuity of $J(t, s)$ in $B(X)$ is deduced from (1.3), since $\|J(t, s)\|_{X}$ is uniformly bounded on $\Delta_{0}$ and $Y$ is dense in $X$; hence $\Xi J(t, s)$ is strongly continuous on $\Delta_{0}$ in $B(Z)$, by the strong continuity of $\Xi$. This fact together with (1.4) implies the strong continuity of $S(t) J(t, s)$ on $\Delta_{0}$ in $B(Y, Z)$. Condition (A2) is proved by using condition (S) and the norm continuity of $S(t)$ on $[0, T]$ in $B(Y, Z)$. In a way similar to the argument in the preceding paragraph, (A3) and (A4) are easily checked.

2. Discrete scheme for evolution operators. In this section we construct a difference scheme to get solution operators governed by $\{A(t): t \in$ $[0, T]\}$. For this purpose, conditions (A1) through (A4) are assumed throughout this section.

LEMma 2.1. Let $\left\{t_{i}\right\}_{i=0}^{l}$ be a sequence such that $\left(t_{i}, t_{i-1}\right) \in \Delta_{0}$ for $i=$ $1, \ldots, l$. Let $x_{0} \in Y$ and set $x_{i}=\prod_{j=1}^{i} J\left(t_{j}, t_{j-1}\right) x_{0}$ for $1 \leq i \leq l$. Then $x_{i} \in Y$ for $1 \leq i \leq l$, and there exist $M_{Y} \geq 1$ and $\beta \geq 0$ such that

$$
\left\|x_{i}\right\|_{Y} \leq M_{Y} \exp \left(\beta\left(t_{i}-t_{0}\right)\right)\left\|x_{0}\right\|_{Y}
$$

for $1 \leq i \leq l$.

Proof. Let $1 \leq i \leq l$ and $1 \leq j \leq i$, and set $a_{j}=\left\|x_{j}\right\|_{X}+\left\|S\left(t_{j}\right) x_{j}\right\|_{Z}$. Using (1.4) with $(t, s)=\left(t_{j}, t_{j-1}\right)$ and $y=x_{j-1}$, we find

$$
\begin{aligned}
\left\|\prod_{k=j+1}^{i} \Xi J\left(t_{k}, t_{k-1}\right) S\left(t_{j}\right) x_{j}-\prod_{k=j}^{i} \Xi J\left(t_{k}, t_{k-1}\right) S\left(t_{j-1}\right) x_{j-1}\right\|_{Z} \\
\quad \leq\left(t_{j}-t_{j-1}\right) M\left(\left(\|B\|_{Z} \vee\|C\|_{X, Z}\right) a_{j-1}+\left\|E_{4}\right\|_{Y, Z}\left\|x_{j-1}\right\|_{Y}\right) .
\end{aligned}
$$

Here we have used condition (A1) and the nonexpansiveness of the algebraic homomorphism $\Xi$. By condition (S), the right-hand side of the inequality above is bounded by $\beta\left(t_{j}-t_{j-1}\right) a_{j-1}$, where $\beta=M\left(\left(\|B\|_{Z} \vee\|C\|_{X, Z}\right)+\right.$ $\left.c_{S}^{-1}\left\|E_{4}\right\|_{Y, Z}\right)$. We sum up the inequalities obtained for $j=1, \ldots, i$ and combine the resulting inequality with the estimate $\left\|x_{i}\right\|_{X} \leq M\left\|x_{0}\right\|_{X}$, which follows from (A1). This yields $a_{i} \leq M a_{0}+\sum_{j=1}^{i} \beta\left(t_{j}-t_{j-1}\right) a_{j-1}$. Denote by $b_{i}$ the right-hand side. Then $a_{i} \leq b_{i}$ and

$$
b_{i} \leq\left(1+\beta\left(t_{i}-t_{i-1}\right)\right) b_{i-1} \leq \exp \left(\beta\left(t_{i}-t_{i-1}\right)\right) b_{i-1} ;
$$

hence $a_{i} \leq M \exp \left(\beta\left(t_{i}-t_{0}\right)\right) a_{0}$. The desired estimate (2.1) is obtained by using condition $(\mathrm{S})$.

LEMma 2.2. Let $\left\{t_{i}\right\}_{i=0}^{\infty}$ be a sequence such that $\left(t_{i}, t_{i-1}\right) \in \Delta_{0}$ for $i \geq 1$. Then the limit $\lim _{i \rightarrow \infty} \prod_{l=k+1}^{i} \Xi J\left(t_{l}, t_{l-1}\right) z$ exists in $Z$, for all $z \in Z$ and $k \geq 0$. 
Proof. Let $y \in Y$ and $k \geq 0$ be an integer, and set $x_{i}=\prod_{l=k+1}^{i} J\left(t_{l}, t_{l-1}\right) y$ for $i \geq k+1$, and $x_{k}=y$. By condition (A3) we have

$$
x_{l}=x_{l-1}+\left(t_{l}-t_{l-1}\right)\left(A\left(t_{l}\right) x_{l}+E_{1}\left(t_{l}, t_{l-1}\right) x_{l-1}\right) \quad \text { for } l \geq k+1 .
$$

We use the estimate (2.1) to find

$$
\left\|x_{i}-x_{j}\right\|_{X} \leq\left(t_{i}-t_{j}\right)\left(\|A\|_{Y, X}+\left\|E_{1}\right\|_{Y, X}\right) M_{Y} \exp (\beta T)\|y\|_{Y}
$$

for $i \geq j \geq k+1$. This implies that $\left\{x_{i}\right\}$ is a Cauchy sequence in $X$ because $\left\{t_{i}\right\}_{i=0}^{\infty}$ is an increasing bounded sequence (and so it is convergent). Condition (A1) and the density of $Y$ in $X$ yield that the limit $\lim _{i \rightarrow \infty} \prod_{l=k+1}^{i} J\left(t_{l}, t_{l-1}\right) x$ exists in $X$, for all $x \in X$. The desired claim follows readily from the strong continuity of $\Xi$.

Proposition 2.3. Let $(s, y) \in[0, T) \times Y$ and $\varepsilon>0$. Then there exists a sequence $\left\{t_{i}\right\}_{i=0}^{N}$ which has the following properties:

(i) $s=t_{0}<t_{1}<\ldots<t_{N}=T$.

(ii) $t_{i}-t_{i-1} \leq \varepsilon$ and $\left(t_{i}, t_{i-1}\right) \in \Delta_{0}$ for $i=1, \ldots, N$.

(iii) $x_{i}:=\prod_{l=1}^{i} J\left(t_{l}, t_{l-1}\right) y \in Y$ for $i=1, \ldots, N$.

(iv) $\left\|x_{i}-x_{i-1}-\left(t_{i}-t_{i-1}\right) A\left(t_{i}\right) x_{i}\right\|_{X} \leq\left(t_{i}-t_{i-1}\right) \varepsilon$ for $i=1, \ldots, N$, where $x_{0}=y$.

(v) $\| S\left(t_{i}\right) x_{i}-\Xi J\left(t_{i}, t_{i-1}\right) S\left(t_{i-1}\right) x_{i-1}-$ $\left(t_{i}-t_{i-1}\right)\left(B\left(t_{i}\right) S\left(t_{i}\right)+C\left(t_{i}\right)\right) x_{i} \|_{Z} \leq\left(t_{i}-t_{i-1}\right) \varepsilon$ for $i=1, \ldots, N$.

(vi) $\left\|x_{i}-x_{i-1}\right\|_{Y} \leq \varepsilon$ for $i=1, \ldots, N$.

(vii) $\left\|\left(A(t)-A\left(t_{i-1}\right)\right) x_{i-1}\right\|_{X} \leq \varepsilon$ for $t \in\left[t_{i-1}, t_{i}\right]$ and $i=1, \ldots, N$.

Proof. Set $\left(t_{0}, x_{0}\right)=(s, y)$ and assume that a sequence $\left\{t_{i}\right\}_{i=0}^{k-1}$ has been chosen so that properties (i) through (vii) hold for $0 \leq i \leq k-1$, where $k \geq 1$ is an integer. If $t_{k-1}=T$ then the proof is complete. If $t_{k-1}<T$ then we define $h_{k}$ to be the largest number satisfying the following five conditions:

$$
\begin{gathered}
0 \leq h_{k} \leq \varepsilon \text { and }\left(t_{k-1}+h_{k}, t_{k-1}\right) \in \Delta_{0}, \\
\left\|E_{1}\left(t_{k-1}+h_{k}, t_{k-1}\right) x_{k-1}\right\|_{X} \leq \varepsilon, \\
\left\|E_{2}\left(t_{k-1}+h_{k}, t_{k-1}\right) x_{k-1}\right\|_{Z} \leq \varepsilon, \\
\left\|J\left(t_{k-1}+h_{k}, t_{k-1}\right) x_{k-1}-x_{k-1}\right\|_{Y} \leq \varepsilon, \\
\left\|\left(A(t)-A\left(t_{k-1}\right)\right) x_{k-1}\right\|_{X} \leq \varepsilon \text { for } t \in\left[t_{k-1}, t_{k-1}+h_{k}\right] .
\end{gathered}
$$

Since $x_{k-1} \in Y$, we have $h_{k}>0$ by condition (A2) and our assumption on strong continuity.

Now, we define $t_{k}=t_{k-1}+h_{k}$ and $x_{k}=J\left(t_{k-1}+h_{k}, t_{k-1}\right) x_{k-1}$. Properties (iv) and (v) follow from conditions (A3) and (A4) respectively, by using (2.3) and (2.4). All the other properties are clearly satisfied with $i=k$. We have only to prove that there exists an integer $N$ such that $t_{N}=T$. Assume to the contrary that $t_{i}<T$ for all $i \geq 1$, and put $\bar{t}=\lim _{i \rightarrow \infty} t_{i}$. We first 
show that the sequence $\left\{x_{i}\right\}_{i=1}^{\infty}$ is convergent in $Y$ as $i \rightarrow \infty$. Using the estimate (2.1) we have, by (iv),

$$
\left\|x_{i}-x_{i-1}\right\|_{X} \leq\left(\|A\|_{Y, X} M_{Y} \exp (\beta T)\|y\|_{Y}+\varepsilon\right)\left(t_{i}-t_{i-1}\right)
$$

for $i \geq 1$, which implies that $\left\{x_{i}\right\}_{i=1}^{\infty}$ is a Cauchy sequence in $X$. Let $k<i$ and $k+1 \leq j \leq i$. By (v) we have

$$
\begin{aligned}
& \| \prod_{l=j+1}^{i} \Xi J\left(t_{l}, t_{l-1}\right) S\left(t_{j}\right) x_{j}-\prod_{l=j}^{i} \Xi J\left(t_{l}, t_{l-1}\right) S\left(t_{j-1}\right) x_{j-1} \|_{Z} \\
& \leq\left(t_{j}-t_{j-1}\right) M\left(\left(\|B\|_{Z} \vee\|C\|_{X, Z}\right) C_{S}\left\|x_{i}\right\|_{Y}+\varepsilon\right) .
\end{aligned}
$$

Here we have used the nonexpansiveness of $\Xi$ and condition (A1). Since $\left\|x_{i}\right\|_{Y} \leq M_{Y} \exp (\beta T)\|y\|_{Y}$ for $i \geq 1$, we find

$$
\begin{aligned}
\| S\left(t_{i}\right) x_{i}- & \prod_{l=k+1}^{i} \Xi J\left(t_{l}, t_{l-1}\right) S\left(t_{k}\right) x_{k} \|_{Z} \\
& \leq\left(t_{i}-t_{k}\right) M\left(\left(\|B\|_{Z} \vee\|C\|_{X, Z}\right) C_{S} M_{Y} \exp (\beta T)\|y\|_{Y}+\varepsilon\right),
\end{aligned}
$$

by summing up the inequalities above for $k+1 \leq j \leq i$. By Lemma 2.2 we have

$$
\begin{aligned}
\limsup _{i, j \rightarrow \infty} \| & S\left(t_{i}\right) x_{i}-S\left(t_{j}\right) x_{j} \|_{Z} \\
& \leq 2\left(\bar{t}-t_{k}\right) M\left(\left(\|B\|_{Z} \vee\|C\|_{X, Z}\right) C_{S} M_{Y} \exp (\beta T)\|y\|_{Y}+\varepsilon\right),
\end{aligned}
$$

which tends to zero as $k \rightarrow \infty$. We therefore deduce from the norm continuity of $S(t)$ on $[0, T]$ in $B(Y, Z)$ and condition (S) that the sequence $\left\{x_{i}\right\}_{i=1}^{\infty}$ is convergent in $Y$ as $i \rightarrow \infty$.

Now, we turn to the proof of the desired claim. For $i \geq 1$ we set $\gamma_{i}=$ $\bar{t}-t_{i-1}$. Clearly, $h_{i}<\gamma_{i}$ for all $i \geq 1$. Moreover, $\lim _{i \rightarrow \infty} \gamma_{i}=0$, which implies that $0 \leq \gamma_{i} \leq \varepsilon$ and $\left(t_{i-1}+\gamma_{i}, t_{i-1}\right) \in \Delta_{0}$ for sufficiently large $i$. By our assumption on strong continuity and the convergence of $\left\{x_{i}\right\}$ in $Y$, there exists an integer $i_{0} \geq 1$ such that if $i \geq i_{0}$ then conditions (2.2) through (2.5) are satisfied with $h_{k}$ and $k$ replaced by $\gamma_{i}$ and $i$. We then deduce from the definition of $h_{i}$ that for each $i \geq i_{0}$ there exists $\widehat{t}_{i} \in\left[t_{i-1}, t_{i-1}+\gamma_{i}\right]$ such that $\left\|\left(A\left(\widehat{t}_{i}\right)-A\left(t_{i-1}\right)\right) x_{i-1}\right\|_{X}>\varepsilon$. Since $\bar{t}=\lim _{i \rightarrow \infty} \widehat{t}_{i}$, the strong continuity of $A(t)$ on $[0, T]$ in $B(Y, X)$ implies $\varepsilon \leq 0$, which is a contradiction.

3. A characterization of evolution operators. The main theorem of this paper is given by

TheOREM 3.1. Let $\{A(t): t \in[0, T]\}$ be a family of closed linear operators in $X$ satisfying condition (A). Then there exists a unique solution operator $\{U(t, s):(t, s) \in \Delta\}$ on $X$, satisfying $\|U(t, s)\|_{X} \leq M$ for $(t, s) \in \Delta$, governed by $\{A(t): t \in[0, T]\}$ if and only if there exist $\varepsilon_{0}>0$ and a family 
$\{J(t, s)\}$ in $B(X)$ defined on the set $\Delta_{0}=\left\{(t, s) \in \Delta:|t-s| \leq \varepsilon_{0}\right\}$ such that conditions (A1) through (A4) of Proposition 1.2 are satisfied.

It has already been shown that conditions (A1) through (A4) are necessary for the existence of a solution operator governed by $\{A(t): t \in[0, T]\}$. Now, to prove the sufficiency assume that conditions (A1) through (A4) are satisfied.

Let $P=\left\{0=s_{0}<s_{1}<\ldots<s_{K}=T\right\}$ be a partition of $[0, T]$ such that $\left(s_{i}, s_{i-1}\right) \in \Delta_{0}$ for $i=1, \ldots, K$. For such a partition we define an operator $U(t, s ; P)$ on $\Delta$ by

$$
U(t, s ; P)=\prod_{l=p+1}^{i} J\left(s_{l}, s_{l-1}\right)
$$

whenever $t \in\left(s_{i-1}, s_{i}\right] \cap[0, T]$ and $s \in\left(s_{p-1}, s_{p}\right] \cap[0, T]$. Here and subsequently, we set $s_{-1}=-\infty$ for convenience. If $F$ is an operator-valued function on $[0, T]$ then we define a step function $F(t ; P)$ by

$$
F(t ; P)=F\left(s_{i}\right) \quad \text { for } t \in\left(s_{i-1}, s_{i}\right] \cap[0, T] \text { and } i=1, \ldots, K .
$$

Lemma 3.2. Let $(s, y) \in[0, T) \times Y$ and $\varepsilon>0$. Let $\left\{\left(t_{i}, x_{i}\right)\right\}_{i=0}^{N}$ be a sequence in $[s, T] \times Y$ as in Proposition 2.3, and define a step function $u:[s, T] \rightarrow Y$ by

$$
u(t)= \begin{cases}x_{0} & \text { for } t=s, \\ x_{i} & \text { for } t \in\left(t_{i-1}, t_{i}\right] \text { and } i=1, \ldots, N .\end{cases}
$$

If $P=\left\{0=s_{0}<s_{1}<\ldots<s_{K}=T\right\}$ is a partition of $[0, T]$ satisfying

$$
|P|:=\max _{1 \leq k \leq K}\left(s_{k}-s_{k-1}\right) \leq \min _{1 \leq i \leq N}\left(t_{i}-t_{i-1}\right),
$$

then

$$
\begin{aligned}
\| u(t)-U(t, s ; P) & y \|_{X} \\
\leq & \left\{C_{S}+M\left(\left(4+3\|A\|_{Y, X}+\left\|E_{3}\right\|_{Y, X}\right) T+C_{S}\right)\right\} \varepsilon \\
& +M T \max _{1 \leq i \leq N} \varrho\left(x_{i-1} ;|P|\right)
\end{aligned}
$$

for $t \in[s, T]$, where for each $w \in Y$ and $r \geq 0, \varrho(w ; r)$ is defined by

$$
\varrho(w ; r)=\sup \left\{\left\|E_{3}(t, s) w\right\|_{X}:|t-s| \leq r \text { and }(t, s) \in \Delta_{0}\right\} .
$$

Proof. Since $P$ is a partition of $[0, T]$, there exists $p \in\{0,1, \ldots, K\}$ such that $t_{0}=s \in\left(s_{p-1}, s_{p}\right] \cap[0, T]$. We note that $p \neq K$ by (3.1). For $k=p+1, \ldots, K$ we define $y_{k}=J\left(s_{k}, s_{k-1}\right) y_{k-1}$ and $y_{p}=x_{0}=y$. Moreover, we use an auxiliary function $v:[s, T] \rightarrow Y$ defined by

$$
\begin{aligned}
v(t)=x_{i-1}+\left(t-t_{i-1}\right)\left(x_{i}-x_{i-1}\right) / & \left(t_{i}-t_{i-1}\right) \\
& \text { for } t \in\left[t_{i-1}, t_{i}\right] \text { and } i=1, \ldots, N .
\end{aligned}
$$


If $s_{k} \in\left[t_{i-1}, t_{i}\right]$ for some $i \in\{1, \ldots, N\}$ then we have, for $l=i-1$ and $i$,

$$
\left\|v\left(s_{k}\right)-x_{l}\right\|_{Y}=\left|s_{k}-t_{l}\right| \cdot\left\|x_{i}-x_{i-1}\right\|_{Y} /\left(t_{i}-t_{i-1}\right) \leq \varepsilon .
$$

Here we have used (vi) of Proposition 2.3. Since $s_{p} \in\left[t_{0}, t_{1}\right]$ we have, by (3.3) and condition (S),

$$
\left\|v\left(s_{p}\right)-y_{p}\right\|_{X} \leq C_{S} \varepsilon
$$

For $k=p+1, \ldots, K$ we set

$$
z_{k}=v\left(s_{k}\right)-v\left(s_{k-1}\right)-\left(s_{k}-s_{k-1}\right)\left(A\left(s_{k-1}\right)+E_{3}\left(s_{k}, s_{k-1}\right)\right) v\left(s_{k-1}\right) .
$$

Since $v\left(s_{k}\right)=J\left(s_{k}, s_{k-1}\right) v\left(s_{k-1}\right)+z_{k}$ by $(1.3)$, we have

$$
v\left(s_{k}\right)-y_{k}=J\left(s_{k}, s_{k-1}\right)\left(v\left(s_{k-1}\right)-y_{k-1}\right)+z_{k} ;
$$

hence it is shown inductively that

$$
v\left(s_{k}\right)-y_{k}=\prod_{l=p+1}^{k} J\left(s_{l}, s_{l-1}\right)\left(v\left(s_{p}\right)-y_{p}\right)+\sum_{j=p+1}^{k} \prod_{l=j+1}^{k} J\left(s_{l}, s_{l-1}\right) z_{j}
$$

for $k=p, \ldots, K$. We need to estimate $z_{k}$ for $k=p+1, \ldots, K$. So, let $k \in\{p+1, \ldots, K\}$. By (3.1), either (i) there exists $i \in\{1, \ldots, N\}$ such that $s_{k-1}, s_{k} \in\left[t_{i-1}, t_{i}\right]$, or else (ii) there exists $i \in\{1, \ldots, N-1\}$ such that $s_{k-1} \in\left[t_{i-1}, t_{i}\right]$ and $s_{k} \in\left[t_{i}, t_{i+1}\right]$. In both cases we have

Indeed, since

$$
\begin{array}{r}
\left\|\left(x_{i}-x_{i-1}\right) /\left(t_{i}-t_{i-1}\right)-\left(A\left(s_{k-1}\right)+E_{3}\left(s_{k}, s_{k-1}\right)\right) v\left(s_{k-1}\right)\right\|_{X} \\
\leq\left(3+2\|A\|_{Y, X}+\left\|E_{3}\right\|_{Y, X}\right) \varepsilon+\max _{1 \leq i \leq N} \varrho\left(x_{i-1} ;|P|\right) .
\end{array}
$$

$$
\begin{aligned}
A\left(t_{i}\right) x_{i}-\left(A\left(s_{k-1}\right)+E_{3}\left(s_{k}, s_{k-1}\right)\right) v\left(s_{k-1}\right) & \\
= & A\left(t_{i}\right)\left(x_{i}-x_{i-1}\right)+\left(A\left(t_{i}\right)-A\left(t_{i-1}\right)\right) x_{i-1}+\left(A\left(t_{i-1}\right)-A\left(s_{k-1}\right)\right) x_{i-1} \\
\quad & +\left(A\left(s_{k-1}\right)+E_{3}\left(s_{k}, s_{k-1}\right)\right)\left(x_{i-1}-v\left(s_{k-1}\right)\right)-E_{3}\left(s_{k}, s_{k-1}\right) x_{i-1},
\end{aligned}
$$

we have, by (vi) and (vii) of Proposition 2.3 and (3.3),

$$
\begin{aligned}
\| A\left(t_{i}\right) x_{i}-( & \left.A\left(s_{k-1}\right)+E_{3}\left(s_{k}, s_{k-1}\right)\right) v\left(s_{k-1}\right) \|_{X} \\
& \leq\left(2+2\|A\|_{Y, X}+\left\|E_{3}\right\|_{Y, X}\right) \varepsilon+\max _{1 \leq i \leq N} \varrho\left(x_{i-1} ;|P|\right) .
\end{aligned}
$$

This estimate and (iv) of Proposition 2.3 together imply (3.6).

We begin by considering the case of (ii). By the definition of $v, z_{k}$ is written as

$$
\begin{aligned}
& \left(s_{k}-t_{i}\right)\left(\left(x_{i+1}-x_{i}\right) /\left(t_{i+1}-t_{i}\right)-\left(A\left(s_{k-1}\right)+E_{3}\left(s_{k}, s_{k-1}\right)\right) v\left(s_{k-1}\right)\right) \\
& \quad+\left(t_{i}-s_{k-1}\right)\left(\left(x_{i}-x_{i-1}\right) /\left(t_{i}-t_{i-1}\right)-\left(A\left(s_{k-1}\right)+E_{3}\left(s_{k}, s_{k-1}\right)\right) v\left(s_{k-1}\right)\right) .
\end{aligned}
$$

or

$$
\begin{aligned}
\left(v\left(s_{k}\right)-v\left(t_{i}\right)\right)+\left(v\left(t_{i}\right)-v\left(s_{k-1}\right)\right) & \\
& -\left(s_{k}-s_{k-1}\right)\left(A\left(s_{k-1}\right)+E_{3}\left(s_{k}, s_{k-1}\right)\right) v\left(s_{k-1}\right),
\end{aligned}
$$


Since $\left(x_{i+1}-x_{i}\right) /\left(t_{i+1}-t_{i}\right)-A\left(t_{i}\right) x_{i}=\left(x_{i+1}-x_{i}\right) /\left(t_{i+1}-t_{i}\right)-A\left(t_{i+1}\right) x_{i+1}+$ $A\left(t_{i+1}\right)\left(x_{i+1}-x_{i}\right)+\left(A\left(t_{i+1}\right)-A\left(t_{i}\right)\right) x_{i}$, we have, by (iv), (vi) and (vii) of Proposition 2.3 and (3.7),

$$
\begin{array}{r}
\left\|\left(x_{i+1}-x_{i}\right) /\left(t_{i+1}-t_{i}\right)-\left(A\left(s_{k-1}\right)+E_{3}\left(s_{k}, s_{k-1}\right)\right) v\left(s_{k-1}\right)\right\|_{X} \\
\leq\left(4+3\|A\|_{Y, X}+\left\|E_{3}\right\|_{Y, X}\right) \varepsilon+\max _{1 \leq i \leq N} \varrho\left(x_{i-1} ;|P|\right) .
\end{array}
$$

By this estimate together with (3.6) we find

$$
\begin{aligned}
\left\|z_{k}\right\|_{X} \leq & \left(4+3\|A\|_{Y, X}+\left\|E_{3}\right\|_{Y, X}\right)\left(s_{k}-s_{k-1}\right) \varepsilon \\
& +\left(s_{k}-s_{k-1}\right) \max _{1 \leq i \leq N} \varrho\left(x_{i-1} ;|P|\right) .
\end{aligned}
$$

In the case of (i), we deduce from (3.6) that (3.8) is also valid, because $z_{k}=\left(s_{k}-s_{k-1}\right)\left(\left(x_{i}-x_{i-1}\right) /\left(t_{i}-t_{i-1}\right)-\left(A\left(s_{k-1}\right)+E_{3}\left(s_{k}, s_{k-1}\right)\right) v\left(s_{k-1}\right)\right)$. We use condition (A1), (3.4) and (3.8) to estimate the quantity (3.5). This yields

$$
\begin{aligned}
\left\|v\left(s_{k}\right)-y_{k}\right\|_{X} \leq & M\left\{\left(\left(4+3\|A\|_{Y, X}+\left\|E_{3}\right\|_{Y, X}\right)\left(s_{k}-s_{p}\right)+C_{S}\right) \varepsilon\right. \\
& \left.+\left(s_{k}-s_{p}\right) \max _{1 \leq i \leq N} \varrho\left(x_{i-1} ;|P|\right)\right\}
\end{aligned}
$$

for $k=p, \ldots, K$.

Now, we turn to the proof of (3.2). Let $t \in(s, T]$. Then there exists $k \in$ $\{1, \ldots, K\}$ such that $t \in\left(s_{k-1}, s_{k}\right]$. Since $s \in\left(s_{p-1}, s_{p}\right]$ we have $U(t, s ; P) y=$ $y_{k}$. If $s_{k} \in\left(t_{i-1}, t_{i}\right]$ for some $i \in\{1, \ldots, N\}$ then $u(t)=x_{i-1}$ or $x_{i}$. It follows from (3.3) that $\left\|v\left(s_{k}\right)-u(t)\right\|_{X} \leq C_{S} \varepsilon$. The desired estimate (3.2) is obtained by combining this estimate and (3.9).

Proof of Theorem 3.1. Let $x \in X,(s, y) \in[0, T) \times Y$ and $\varepsilon>0$. If $P$ and $\widehat{P}$ are two partitions of $[0, T]$ then by Lemma 3.2 we have

$\limsup \left(\sup \left\{\|U(t, s ; P) x-U(t, s ; \widehat{P}) x\|_{X}: t \in[s, T]\right\}\right)$ $|P|,|\widehat{P}| \rightarrow 0$

$$
\leq 2\left\{C_{S}+M\left(\left(4+3\|A\|_{Y, X}+\left\|E_{3}\right\|_{Y, X}\right) T+C_{S}\right)\right\} \varepsilon+2 M\|x-y\|_{X} .
$$

This implies that for $x \in X$ and $(t, s) \in \Delta$, the limit

$$
U(t, s) x=\lim _{|P| \rightarrow 0} U(t, s ; P) x
$$

exists independently of the choice of the partition $P$ of $[0, T]$. Property (E1) is satisfied because $U(t, t ; P)=I$ and $U(t, r ; P) U(r, s ; P)=U(t, s ; P)$ for $(t, r),(r, s) \in \Delta$.

To prove (E2), let $w \in Y$ and $P=\left\{0=s_{0}<s_{1}<\ldots<s_{K}=T\right\}$ be a partition of $[0, T]$ such that $\left(s_{i}, s_{i-1}\right) \in \Delta_{0}$ for $i=1, \ldots, K$. For $0 \leq k \leq j<i \leq K$ we find, by using (1.1) with $(t, s)=\left(s_{p}, s_{p-1}\right)$ and 
$y=\prod_{l=k+1}^{p-1} J\left(s_{l}, s_{l-1}\right) w$,

$$
\begin{aligned}
& \| \prod_{l=k+1}^{p} J\left(s_{l}, s_{l-1}\right) w- \prod_{l=k+1}^{p-1} J\left(s_{l}, s_{l-1}\right) w \|_{X} \\
& \leq\left(s_{p}-s_{p-1}\right)\left(\|A\|_{Y, X}+\left\|E_{1}\right\|_{Y, X}\right) M_{Y} \exp (\beta T)\|w\|_{Y}
\end{aligned}
$$

for $p=j+1, \ldots, i$. Summing up these inequalities we have

$$
\begin{aligned}
& \|U(t, s ; P) w-U(\widehat{t}, s ; P) w\|_{X} \\
& \quad \leq M_{Y} \exp (\beta T)(|t-\widehat{t}|+2|P|)\left(\|A\|_{Y, X}+\left\|E_{1}\right\|_{Y, X}\right)\|w\|_{Y}
\end{aligned}
$$

for $(t, s),(\hat{t}, s) \in \Delta$. In a way similar to the derivation of (3.10), we use (1.3) with $(t, s)=\left(s_{k}, s_{k-1}\right)$ and $y=w$ to obtain

$\|U(t, s ; P) w-U(t, \widehat{s} ; P) w\|_{X} \leq M(|s-\widehat{s}|+2|P|)\left(\|A\|_{Y, X}+\left\|E_{3}\right\|_{Y, X}\right)\|w\|_{Y}$ for $(t, s),(t, \widehat{s}) \in \Delta$. This estimate and (3.10) together imply the strong continuity of $U(t, s)$ on $\Delta$ in $B(X)$.

Now, to check properties (E3) through (E5) let $(s, y) \in[0, T) \times Y$ and $\varepsilon>0$. If $s>0$ then we choose a sequence $\left\{s_{k}^{\varepsilon}\right\}_{k=0}^{l_{\varepsilon}}$ such that $0=s_{0}^{\varepsilon}<s_{1}^{\varepsilon}<$ $\ldots<s_{l_{\varepsilon}}^{\varepsilon}=s$ and that $s_{k}^{\varepsilon}-s_{k-1}^{\varepsilon} \leq \varepsilon$ and $\left(s_{k}^{\varepsilon}, s_{k-1}^{\varepsilon}\right) \in \Delta_{0}$ for $k=1, \ldots, l_{\varepsilon}$. We denote by $P_{\varepsilon}=\left\{0=s_{0}^{\varepsilon}<s_{1}^{\varepsilon}<\ldots<s_{K_{\varepsilon}}^{\varepsilon}=T\right\}$ the partition of $[0, T]$ which is constructed by appending points $t_{i}(i=1, \ldots, N)$ in $[s, T]$ satisfying properties (i) through (vii) of Proposition 2.3 to the sequence $\left\{s_{k}^{\varepsilon}\right\}$ above. We then deduce from the first part of the proof of the theorem that

$$
U(t, r) x=\lim _{\varepsilon \downarrow 0} U\left(t, r ; P_{\varepsilon}\right) x
$$

for $x \in X$ and $(t, r) \in \Delta$. By (v) of Proposition 2.3 we have

$$
\begin{aligned}
& \| \prod_{l=j+1}^{i} \Xi J\left(t_{l}, t_{l-1}\right) S\left(t_{j}\right) x_{j}-\prod_{l=j}^{i} \Xi J\left(t_{l}, t_{l-1}\right) S\left(t_{j-1}\right) x_{j-1} \\
& \quad-\left(t_{j}-t_{j-1}\right) \prod_{l=j+1}^{i} \Xi J\left(t_{l}, t_{l-1}\right)\left(B\left(t_{j}\right) S\left(t_{j}\right)+C\left(t_{j}\right)\right) x_{j} \|_{Z} \leq M\left(t_{j}-t_{j-1}\right) \varepsilon
\end{aligned}
$$

for $1 \leq j \leq i$. Summing up these inequalities we find

$$
\begin{aligned}
& \| S\left(t ; P_{\varepsilon}\right) U\left(t, s ; P_{\varepsilon}\right) y-\Xi U\left(t, s ; P_{\varepsilon}\right) S(s) y \\
& -\int_{s}^{t_{i}} \Xi U\left(t, \sigma ; P_{\varepsilon}\right)\left(B\left(\sigma ; P_{\varepsilon}\right) S\left(\sigma ; P_{\varepsilon}\right)+C\left(\sigma ; P_{\varepsilon}\right)\right) U\left(\sigma, s ; P_{\varepsilon}\right) y d \sigma \|_{Z} \leq M\left(t_{i}-s\right) \varepsilon
\end{aligned}
$$

for $t \in\left(t_{i-1}, t_{i}\right] \cap[0, T]$ where $i \geq 0$ is an integer. On the other hand, by a fixed point argument there exists a unique strongly continuous family $\{V(t, r):(t, r) \in \Delta\}$ in $B(Y, Z)$ satisfying the Volterra type integral 
equation

$$
V(t, r) w=\Xi U(t, r) S(r) w+\int_{r}^{t} \Xi U(t, \sigma)(B(\sigma) V(\sigma, r) w+C(\sigma) U(\sigma, r) w) d \sigma
$$

for $w \in Y$ and $(t, r) \in \Delta$. Now, set

$$
\varphi(t)=\limsup _{\varepsilon \downarrow 0}\left\|S\left(t ; P_{\varepsilon}\right) U\left(t, s ; P_{\varepsilon}\right) y-V(t, s) y\right\|_{Z}
$$

for $t \in[s, T]$. Then $\varphi(t) \leq M\|B\|_{Z} \int_{s}^{t} \varphi(\sigma) d \sigma$ for $t \in[s, T]$. Here we have used the fact that $\lim _{\varepsilon \downarrow 0} \Xi U\left(t, r ; P_{\varepsilon}\right) z=\Xi U(t, r) z$ in $Z$, for $z \in Z$ and $(t, r) \in \Delta$, which follows from (3.11) and the strong continuity of $\Xi$. An application of Gronwall's inequality gives $\varphi(t)=0$ for $t \in[s, T]$. This fact together with the norm continuity of $S(t)$ implies that for $t \in[s, T]$, $\lim _{\varepsilon \downarrow 0} U\left(t, s ; P_{\varepsilon}\right) y=U(t, s) y$ in $Y$ and $S(t) U(t, s) y=V(t, s) y$. Properties (E3) and (E5) are thus checked.

To prove (E4), we sum up (iv) of Proposition 2.3 from $i=1$ to $i=k$. This yields

$$
\left\|U\left(t, s ; P_{\varepsilon}\right) y-y-\sum_{i=1}^{k} \int_{t_{i-1}}^{t_{i}} A\left(\sigma ; P_{\varepsilon}\right) U\left(\sigma, s ; P_{\varepsilon}\right) y d \sigma\right\|_{X} \leq\left(t_{k}-s\right) \varepsilon
$$

for $t \in\left(t_{k-1}, t_{k}\right] \cap[0, T]$ where $k \geq 0$ is an integer. The desired property (E4) is obtained by taking the limit as $\varepsilon \downarrow 0$.

Finally, we give an extension of [11, Main Theorem] and [6, Appendix C].

According to Kato [6] we assume that the family $\{S(t): t \in[0, T]\}$ also has the property that for $y \in Y, S(t) y$ is differentiable on $[0, T]$ in $Z$ and

$$
\frac{d}{d t} S(t) y=Q(t) S(t) y+R(t) y \quad \text { for } t \in[0, T],
$$

where $\{Q(t): t \in[0, T]\}$ is a strongly continuous family in $B(Z)$ and $\{R(t)$ : $t \in[0, T]\}$ is a strongly continuous family in $B(X, Z)$. (The norm continuity of $S(t)$ on $[0, T]$ in $B(Y, Z)$ follows automatically from (3.12).)

We make the following assumption on the stability condition, which was proposed in [11] from the viewpoint of finite difference approximations.

(H1) There exist $M \geq 1$ and $\lambda_{0}>0$ such that $\left(I-\left(t_{i}-t_{i-1}\right) A\left(t_{i}\right)\right)^{-1} \in$ $B(X)$ and $\left\|\prod_{i=1}^{k}\left(I-\left(t_{i}-t_{i-1}\right) A\left(t_{i}\right)\right)^{-1}\right\|_{X} \leq M$ for every finite sequence $\left\{t_{i}\right\}_{i=0}^{k}$ with $0 \leq t_{0}<t_{1}<\ldots<t_{k} \leq T$ and $t_{i}-t_{i-1} \leq \lambda_{0}$ for $i=1, \ldots, k$.

Remark. By Proposition 1.3, condition (H1) is equivalent to the following condition: There exist $M \geq 1, \lambda_{0}>0$ and a family $\left\{N_{t}(\cdot): t \in[0, T]\right\}$ of norms in $X$ such that

(i) $\|x\|_{X} \leq N_{t}(x) \leq M\|x\|_{X}$ for $t \in[0, T]$ and $x \in X$; 
(ii) $N_{t}\left((I-\lambda A(t))^{-1} x\right) \leq N_{t-\lambda}(x)$ for $t \in(0, T], \lambda \in\left(0, \lambda_{0} \wedge t\right]$ and $x \in X$.

The following condition corresponds to the "intertwining condition" in the sense of Kato [6]:

(H2) For $t \in(0, T]$ and $\lambda \in\left(0, \lambda_{0} \wedge t\right],(I-\lambda A(t))^{-1}(Y) \subset Y$ and

$$
\begin{aligned}
S(t)(I-\lambda A(t))^{-1} y & \\
& =\Xi(I-\lambda A(t))^{-1}\left(S(t) y+\lambda P(t) S(t)(I-\lambda A(t))^{-1} y\right)
\end{aligned}
$$

for $y \in Y$, where $\{P(t): t \in[0, T]\}$ is a strongly continuous family in $B(Z)$.

COROLlaRY 3.3. Under hypotheses (H1) and (H2), there exists a unique solution operator on $X$ governed by $\{A(t): t \in[0, T]\}$.

To prove Corollary 3.3 we set $J(t, s)=(I-(t-s) A(t))^{-1}$ for $(t, s) \in \Delta$ with $0<t-s \leq \lambda_{0}$, and $J(t, t)=I$ for $t \in[0, T]$, and prove the strong continuity of $J(t, s)$ in $B(Y)$ in the following:

LemmA 3.4. Let $\varepsilon_{0}=\lambda_{0} \wedge\left(1 /\left(2 M\|P\|_{Z}\right)\right)$ and set $\Delta_{0}=\{(t, s) \in \Delta$ : $\left.|t-s| \leq \varepsilon_{0}\right\}$. Then the following assertions hold:

(i) The function $(t, s) \mapsto \Xi J(t, s)$ is strongly continuous on $\Delta_{0}$ in $B(Z)$.

(ii) The function $(t, s) \mapsto J(t, s)$ is strongly continuous on $\Delta_{0}$ in $B(Y)$.

Proof. Let $\left(t_{0}, s_{0}\right),(t, s) \in \Delta_{0}$ and $y \in Y$. Since $J(t, s) y-J\left(t_{0}, s_{0}\right) y=$ $J(t, s)\left\{(t-s) A(t)-\left(t_{0}-s_{0}\right) A\left(t_{0}\right)\right\} J\left(t_{0}, s_{0}\right) y$, we deduce from the strong continuity of $A(t)$ on $[0, T]$ in $B(Y, X)$ that $J(t, s)$ is strongly continuous on $\Delta_{0}$ in $B(X)$. The strong continuity of $\Xi$ implies (i). By (3.13) we have

$$
\begin{aligned}
S(t) J(t, s) & y-S\left(t_{0}\right) J\left(t_{0}, s_{0}\right) y \\
= & \Xi J(t, s) S(t) y-\Xi J\left(t_{0}, s_{0}\right) S\left(t_{0}\right) y \\
& +\left((t-s) \Xi J(t, s) P(t)-\left(t_{0}-s_{0}\right) \Xi J\left(t_{0}, s_{0}\right) P\left(t_{0}\right)\right) S\left(t_{0}\right) J\left(t_{0}, s_{0}\right) y \\
& +(t-s) \Xi J(t, s) P(t)\left(S(t) J(t, s) y-S\left(t_{0}\right) J\left(t_{0}, s_{0}\right) y\right) .
\end{aligned}
$$

Since $(t-s)\|\Xi J(t, s) P(t)\|_{Z} \leq(t-s) M\|P\|_{Z}<1$, it follows from (i) that $S(t) J(t, s)$ is strongly continuous on $\Delta_{0}$ in $B(Y, Z)$. By using condition $(\mathrm{S})$, assertion (ii) is deduced from the fact above together with the norm continuity of $S(t)$ on $[0, T]$ in $B(Y, Z)$.

Proof of Corollary 3.3. To apply Theorem 3.1 we prove that conditions (A1) through (A4) are satisfied. Condition (A1) is a direct consequence of (H1), and condition (A2) follows from (ii) of Lemma 3.4 and (H2). It is easily seen that (A3) is satisfied with $E_{1}(t, s)=0$ for $(t, s) \in \Delta_{0}$.

To check (A4), let $B(t)=P(t)+Q(t)$ and $C(t)=R(t)$ for $t \in[0, T]$. Clearly $\{B(t): t \in[0, T]\}$ and $\{C(t): t \in[0, T]\}$ are strongly continu- 
ous families on $[0, T]$ in $B(Z)$ and $B(X, Z)$, respectively. Equation (1.2) is satisfied with $E_{2}: \Delta_{0} \rightarrow B(Y, Z)$ defined by

$$
\begin{aligned}
E_{2}(t, s) y= & \frac{1}{t-s} \int_{s}^{t} \Xi J(t, s)(Q(r) S(r) y+R(r) y) d r \\
& -(B(t) S(t)+C(t)) J(t, s) y+\Xi J(t, s) P(t) S(t) J(t, s) y
\end{aligned}
$$

for $(t, s) \in \Delta_{0}$ with $t \neq s$, and $E_{2}(t, t)=0$ for $t \in[0, T]$.

\section{References}

[1] J. R. Dorroh, A simplified proof of a theorem of Kato on linear evolution equations, J. Math. Soc. Japan 27 (1975), 474-478.

[2] J. A. Goldstein, Semigroups of Linear Operators and Applications, Oxford Univ. Press, New York, 1985.

[3] J. V. Herod and R. W. McKelvey, A Hille-Yosida theory for evolutions, Israel J. Math. 36 (1980), 13-40.

[4] S. Ishii, Linear evolution equations $d u / d t+A(t) u=0$ : a case where $A(t)$ is strongly uniform-measurable, J. Math. Soc. Japan 34 (1982), 413-424.

[5] T. Kato, Linear evolution equations of "hyperbolic" type, J. Fac. Sci. Univ. Tokyo 17 (1970), 241-258.

[6] -, Abstract evolution equations, linear and quasilinear, revisited, in: Lecture Notes in Math. 1540, Springer, 1993, 103-125.

[7] K. Kobayasi, On a theorem for linear evolution equations of hyperbolic type, J. Math. Soc. Japan 31 (1979), 647-654.

[8] Y. Kōmura, On linear evolution operators in reflexive Banach spaces, J. Fac. Sci. Univ. Tokyo 17 (1970), 529-542.

[9] A. Pazy, Semigroups of Linear Operators and Applications to Partial Differential Equations, Springer, New York, 1983.

[10] H. Tanabe, Functional Analytic Methods for Partial Differential Equations, Dekker, New York, 1997.

[11] N. Tanaka, Generation of linear evolution operators, Proc. Amer. Math. Soc. 128 (2000), 2007-2015.

[12] A. Yagi, Remarks on proof of a theorem of Kato and Kobayasi on linear evolution equations, Osaka J. Math. 17 (1980), 233-244.

Department of Mathematics

Faculty of Science

Okayama University

Okayama 700-8530, Japan

E-mail: tanaka@math.okayama-u.ac.jp

Received July 21, 2000

Revised version February 8, 2001 